\title{
Application scope of prediction model for interval grey number based on GM $(1,1)$
}

\author{
Lianming Zhao \\ Southwest Jiaotong University,School of Transportation and logistics,Chengdu 610031,China \\ email:cq_zlm@126.com
}

Keywords: Grey theory,Interval grey number prediction model,grey number,Grey degree

Abstract. Prediction models have some modeling conditions and application scope, we can use them to simulate or predict only when the models are satisfies those conditions. In this paper, we study the application scope of prediction model for interval grey number based on GM $(1,1)$ through the mathematical derivation. The reslt shown that when the parameters of prediction model for interval grey number satisfied $\left|a_{s}\right| \geq 2$ or $\mid a_{w} \geq 2$, prediction model for interval grey number is meaningless. The research achievements are of great significance in enrich the grey models and integrate the gray theory with practical issues.

\section{Introduction}

Grey prediction model is an important part of grey theory, mainly for the prediction of grey uncertainty problems. It can use a small amount of valid data and grey uncertain data to reveal the future development trends, through a sequence of accumulated generating system. The grey prediction model has been widely adopt for is not have strict requirements and restrictions about modeling data [1-3]. The current achievements of grey prediction model focus on the modeling mechanism of [4], model parameter optimization of [5], modeling object model extends [6-9] and so on.

Zeng (2010) proposed the concept of grey bound and grey layer of interval grey number sequence, by calculating the area of grey layer and its median lines' coordinate, an interval grey number sequence is changed into a real number sequence without missing any information of the grey number. Then a prediction model for interval grey number based on GM $(1,1)$ is proposed [10], further more, he studied the modeling and application of the prediction model for interval grey numbers and discrete grey numbers when the whitenization weight functions are known.

In fact, Prediction models have some modeling conditions and application scope, we can use them to simulate or predict only when the models are satisfies those conditions. In this paper, we study the application condition of prediction model for interval grey number based on $\operatorname{GM}(1,1)$ in literature [9], in the hope of enriching the grey models and integrate the gray theory with practical issues.

\section{GM $(1,1)$ Model}

Definition 2.1 Let

$$
\begin{gathered}
X^{(0)}=\left(x^{(0)}(1), x^{(0)}(2), \mathrm{L}, x^{(0)}(n)\right), \quad X^{(1)}=\left(x^{(1)}(1), x^{(1)}(2), \mathrm{L}, x^{(1)}(n)\right) \\
x^{(0)}(k)+a x^{(1)}(k)=b
\end{gathered}
$$

Formula $(2.1)$ is the primary form of the $\operatorname{GM}(1,1)[1]$.

$\operatorname{GM}(1,1)$ is defined as follows:

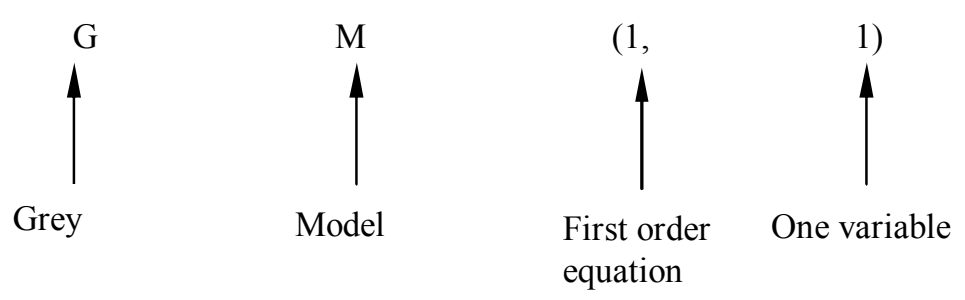


Definition 2.2 $X^{(0)}, X^{(1)}$ are defined as definition 2.1,

$$
Z^{(1)}=\left(z^{(1)}(2), z^{(1)}(3), \mathrm{L}, z^{(1)}(n)\right)
$$

Where $z^{(1)}(k)=\frac{1}{2}\left(x^{(1)}(k)+x^{(1)}(k-1)\right)$, the primary form of GM $(1,1)[1]$ is[1]:

$$
x^{(0)}(k)+a z^{(1)}(k)=b
$$

Theorem 2.1 let $X^{(0)}$ is a nonnegative sequence:

$$
X^{(0)}=\left(x^{(0)}(1), x^{(0)}(2), \mathrm{L}, x^{(0)}(n)\right)
$$

Where $x^{(0)}(k) \geq 0, k=1,2, \mathrm{~L} n ; \quad X^{(1)}$ is a 1-AGO sequence for $X^{(0)}$ :

$$
X^{(1)}=\left(x^{(1)}(1), x^{(1)}(2), \mathrm{L}, x^{(1)}(n)\right)
$$

Where $x^{(1)}(k)=\sum_{i=1}^{k} x^{(0)}(i), \quad k=1,2, \mathrm{~L} n ; Z^{(1)}$ is a neighboring mean sequence for $X^{(1)}$ :

$$
Z^{(1)}=\left(z^{(1)}(2), z^{(1)}(3), \mathrm{L}, z^{(1)}(n)\right)
$$

Where $z^{(1)}(k)=\frac{1}{2}\left(x^{(1)}(k)+x^{(1)}(k-1)\right), \quad k=2,3 \mathrm{~L}, n$.

If $\hat{a}=(a, b)^{T}$ is a Parameter column, and

$$
Y=\left[\begin{array}{c}
x^{(0)}(2) \\
x^{(0)}(3) \\
\mathrm{M} \\
x^{(0)}(n)
\end{array}\right], B=\left[\begin{array}{cc}
-z^{(1)}(2) & 1 \\
-z^{(1)}(3) & 1 \\
\mathrm{M} & \mathrm{M} \\
-z^{(1)}(n) & 1
\end{array}\right]
$$

Then parameters for the least square estimation of GM $(1,1)$ model : $x^{(0)}(k)+a z^{(1)}(k)=b$ is satisfy (2.3):

$$
\hat{a}=\left(B^{T} B\right)^{-1} B^{T} Y
$$

Definition 2.3 let $X^{(0)}$ is a nonnegative sequence, $X^{(1)}$ is a $1-\mathrm{AGO}$ sequence for $X^{(0)}$, $Z^{(1)}$ is a neighboring mean sequence for $X^{(1)},[a, b]^{T}=\left(B^{T} B\right)^{-1} B^{T} Y$. So the winterization equation(Also called Silhouette Formula) [1] of $\operatorname{GM}(1,1) \operatorname{model}: x^{(0)}(k)+a z^{(1)}(k)=b$ is defined by:

$$
\frac{d x^{(1)}}{d t}+a x^{(1)}=b
$$

Theorem 2.2 Let $B, Y, \hat{a}$ are defined as Theorem 2.1, $\hat{a}=[a, b]^{T}=\left(B^{T} B\right)^{-1} B^{T} Y$, then [1]:

1) The solution of winterization equation $\frac{d x^{(1)}}{d t}+a x^{(1)}=b$, also called time responding function is

$$
x^{(1)}(t)=\left(x^{(1)}(1)-\frac{b}{a}\right) e^{-a t}+\frac{b}{a}
$$

2) The Time response sequence of $\operatorname{GM}(1,1)$ model $x^{(0)}(k)+a z^{(1)}(k)=b$ is [1]:

$$
\hat{x}^{(1)}(k+1)=\left(x^{(0)}(1)-\frac{b}{a}\right) e^{-a k}+\frac{b}{a} ; \quad k=1,2, \mathrm{~L} n
$$

3) The reducing value is[1]:

$$
\hat{x}^{(0)}(k+1)=\hat{x}^{(1)}(k+1)-\hat{x}^{(1)}(k)=\left(1-e^{a}\right)\left(x^{(0)}(1)-\frac{b}{a}\right) e^{-a k}, \quad k=1,2, \mathrm{~L} n
$$




\section{Prediction model for interval grey number based on GM $(1,1)$}

The essence of prediction of interval grey number is to predict the upper and lower bounds of the interval grey number, because each element in $X(\otimes)$ is grey number, with a certain grayscale, therefore it can not modeling $X(\otimes)$ in accordance with the traditional GM $(1,1)$ model to real number. The form of upper and lower bounds of the interval grey number is real numbers. A large grayscale of interval grey number means that a large deviation between the upper or lower bounds and the real value. It will influence the correctness of the modeling. And in general, grayscale is unknown, and deficiency in the distribution information. Literature [10] replace the upper and lower bounds with grey band's grey layer during prediction model is building, and weaken the influence of interval grey number's uncertainties in the model.

Let interval grey number sequence is $X(\otimes)=\left(\otimes\left(t_{1}\right), \otimes\left(t_{2}\right), \mathrm{L}, \otimes\left(t_{n}\right)\right), \Delta t_{k}=t_{k}-t_{k-1}=1$, $\otimes\left(t_{k}\right) \in\left[a_{k}, b_{k}\right], \quad k=1,2, \mathrm{~L}, n$, according to literature [10], the area of all gray layer in gray band constitutes a real numbers sequence $S, S=\left(s\left(t_{1}\right), s\left(t_{2}\right), \mathrm{L}, s\left(t_{n-1}\right)\right)$; and the real numbers sequence of the median lines' vertical coordinates in grey layer is $W, W=(w(1), w(2), \mathrm{L}, w(n-1))$, construct $\mathrm{GM}(1$, 1) model of $S$ and $W$ are:

$$
\begin{gathered}
\hat{b}_{n}-\hat{a}_{n}=\frac{2\left(1-e^{a_{s}}\right)\left(s^{(0)}(1)-\frac{b_{s}}{a_{s}}\right) e^{-a_{s}(n-2)}\left[1-\left(-e^{a_{s}}\right)^{n-2}\right]}{1+e^{a_{s}}}+(-1)^{n}\left(b_{2}-a_{2}\right) \\
\hat{a}_{n}+\hat{b}_{n}=\frac{4\left(1-e^{a_{w}}\right)\left(w^{(0)}(1)-\frac{b_{w}}{a_{w}}\right) e^{-a_{w}(n-2)}\left[1-\left(-e^{a_{w}}\right)^{n-2}\right]}{1+e^{a_{w}}}+(-1)^{n}\left(a_{2}+b_{2}\right)
\end{gathered}
$$

Combined (2.8) and (2.9), we can obtain the simulation and prediction model of the upper and lower bounds:

$$
\left\{\begin{array}{l}
\hat{a}_{n}=\frac{-F_{s}-C_{s}+F_{w}+C_{w}}{2} \\
\hat{b}_{n}=\frac{F_{s}+C_{s}+F_{w}+C_{w}}{2}
\end{array}\right.
$$

Of which

$$
\begin{gathered}
F_{s}=\frac{2\left(1-e^{a_{s}}\right)\left(s^{(0)}(1)-\frac{b_{s}}{a_{s}}\right) e^{-a_{s}(n-2)}\left[1-\left(-e^{a_{s}}\right)^{n-2}\right]}{1+e^{a_{s}}} \\
F_{w}=\frac{4\left(1-e^{a_{w}}\right)\left(w^{(0)}(1)-\frac{b_{w}}{a_{w}}\right) e^{-a_{w}(n-2)}\left[1-\left(-e^{a_{w}}\right)^{n-2}\right]}{1+e^{a_{w}}} \\
C_{s}=(-1)^{n}\left(b_{2}-a_{2}\right) \quad C_{w}=(-1)^{n}\left(a_{2}+b_{2}\right)
\end{gathered}
$$

Formula (2.10) is the prediction model for interval grey number based on GM $(1,1)$.

\section{Modeling analysis of prediction model for interval grey number based on GM $(1,1)$}

According to the deducing process and Modeling conditions of traditional GM $(1,1)$ models [11], the parameter calculating equation of formula $(2.10)$ is 


$$
\begin{aligned}
& a_{s}=\frac{\sum_{k=2}^{n} s\left(t_{k}\right) \sum_{k=2}^{n} z_{s}^{(1)}(k)-(n-1) \sum_{k=2}^{n} s\left(t_{k}\right) z_{s}^{(1)}(k)}{(n-1) \sum_{k=2}^{n}\left[z_{s}^{(1)}(k)\right]^{2}-\left[\sum_{k=2}^{n} z_{s}^{(1)}(k)\right]^{2}} \\
& a_{w}=\frac{\sum_{k=2}^{n} w\left(t_{k}\right) \sum_{k=2}^{n} z_{w}^{(1)}(k)-(n-1) \sum_{k=2}^{n} w\left(t_{k}\right) z_{w}^{(1)}(k)}{(n-1) \sum_{k=2}^{n}\left[z_{w}^{(1)}(k)\right]^{2}-\left[\sum_{k=2}^{n} z_{w}^{(1)}(k)\right]^{2}}
\end{aligned}
$$

By formula (2.11) and (2.12),

1) When $(n-1) \sum_{k=2}^{n}\left[z_{s}^{(1)}(k)\right]^{2} \rightarrow\left[\sum_{k=2}^{n} z_{s}^{(1)}(k)\right]^{2}, \quad a_{s} \rightarrow \infty$, formula (x) is meaningless;

2) When $(n-1) \sum_{k=2}^{n}\left[z_{w}^{(1)}(k)\right]^{2} \rightarrow\left[\sum_{k=2}^{n} z_{w}^{(1)}(k)\right]^{2}, \quad a_{w} \rightarrow \infty$, formula (x) is meaningless;

Theorem 2.2, For the parameters of prediction model for interval grey number satisfied $\left|a_{s}\right| \geq 2$ or $\mid a_{w} \geq 2$, prediction model for interval grey number is meaningless.

Proof: The $\mathrm{GM}(1,1)$ model is:

$$
s\left(t_{k}\right)=\left[\frac{1-0.5 a_{s}}{1+0.5 a_{s}}\right]^{k-2}\left[\frac{b_{s}-a_{s} s\left(t_{1}\right)}{1+0.5 a_{s}}\right], \quad k=2,3, \mathrm{~L}, n-1
$$

We can get:

1) When $a_{s}=-2, s\left(t_{k}\right) \rightarrow \infty$;

2) When $a_{s}=2, s\left(t_{k}\right)=0$;

3) When $\left|a_{s}\right|>2, \frac{b_{s}-a_{s} s\left(t_{1}\right)}{1+0.5 a_{s}}$ is constant, and $\frac{1-0.5 a_{s}}{1+0.5 a_{s}}$ subject to change in sign with the different parity of $k$, so $s\left(t_{k}\right)$ will change in sign with the different parity of $k$.

By above knowable, when the parameters of prediction model for interval grey number satisfied $\left|a_{s}\right| \geq 2$ or $\mid a_{w} \geq 2$, prediction model for interval grey number is meaningless, this completes the proof of the theorem.

\section{Conclusions}

Prediction models have some modeling conditions and application scope, we can use them to simulate or predict only when the models are satisfies those conditions. In this paper, we study the application condition of prediction model for interval grey number based on GM $(1,1)$ in literature [9], we draw the following conclusions:

1) $(-\infty,-2] \cup[2, \infty)$ is the Restricted Area of parameter $a_{s}$ of prediction model for interval grey number, when $a_{s} \in(-\infty,-2] \cup[2, \infty)$, prediction model for interval grey number is meaningless.

2) $(-\infty,-2] \cup[2, \infty)$ is the Restricted Area of parameter $a_{w}$ of prediction model for interval grey number, when $a_{w} \in(-\infty,-2] \cup[2, \infty)$, prediction model for interval grey number is meaningless.

The research achievements are of great significance in enrich the grey models and integrate the gray theory with practical issue.

\section{References}

[1] Deng J L. Introduction to grey system theory. The Journal of Grey System (UK), 1989; 1(1):1-24. 
[2] Deng J L. The Primary Methods of Grey Theory. Wuhan: Hua zhong University of Science and Technology Press, 2004: 26-34.

[3] Liu S F, Dang Y G, Fang Z G. Gray system theories and its applications. The fifth Edition. Beijing: Science Press: 2010: 242-246, 155-157, 227.

[4] Xiao X P, Song Z M, Li F. Grey technology foundations and its applications. Beijing: Science Press: 2005

[5] Dang Y G, Liu S F. The GM Models that x (n) be taken as Initial Value. The International Journal of Systems \& Cybernetics, 2004; 33(2): 247-255.

[6] Zeng B, Liu S F, Xie N M. Prediction model of interval grey number based on DGM (1, 1). Journal of Systems Engineering and Electronics, 2010; 21(4):598-603.

[7] Fang Z G, Liu S F. Study on Improvement of Token and Arithmetic of Interval Grey Numbers and Its GM(1,1) Model. Engineering Science 2005; 7(2):57-61.

[8] Meng W, Liu S F, Zeng B. Standardization of interval grey number and research on its prediction modeling and application. Control and Decision, 2012; 27(5): 773-776.

[9] Zeng B, Liu S F, Cui J. Prediction model for interval grey number with known whitenization weight function. Control and Decision, 2010; 25(12):1815-1820.

[10] Zeng B, Liu S F, Xie N M, et al. Prediction model for interval grey number based on grey band and grey layer. Control and Decision, 2010; 25(10): 1585-1588.

[11] Liu S F, Deng J L. The range suitable for GM (1, 1). The Journal of Grey System, 1996; 11(1):131-138. 